\title{
Human embryogenesis in a dish
}

\author{
A 3D culture system recapitulates human development prior to gastrulation and enables transcriptomic studies.
}

$\mathrm{H}$ uman embryogenesis has been modeled in 2D cultures up to days 12-13. "However, these embryos grown in a dish only recapitulate some developmental landmarks and form some simple structures," says Tianqing Li from Kunming University of Science and Technology and from Yunnan Provincial Academy of Science and Technology. Structures or processes such as amniotic epithelium-epiblast separation, basement membrane, secondary yolk sac, anterior visceral endoderm, anterior-posterior polarity initiation and primitive streak anlage are not present in human embryos grown in 2D culture conditions, making it difficult to study spatial self-organization and cell lineages. Thus, better models for studying human pregastrulation embryogenesis are needed.

$\mathrm{Li}$, his colleague Weizhi Ji and their teams addressed this limitation by growing human embryos in a 3D environment. They found that supplementing media used for 2D cultures with 10\% Matrigel allowed human embryos to develop from the blastocyst stage at 5-6 days post-fertilization until day 14 in culture. "Of course, because of a lack of in vivo reference, we cannot guarantee that this $3 \mathrm{D}$ embryo is $100 \%$ similar to an in vivo embryo," says Li. Nevertheless, these embryos appear to recapitulate key morphological processes. The researchers achieved a success rate of about $25 \%$, meaning that a quarter of the embryos survived and developed until day 14 . While it is difficult to compare this rate to human development in vivo, Li says that the pregnancy rate after intrauterine transfer of in vitro-fertilized embryos, which is affected by the uterine environment also, is similar.

The researchers could observe that the blastocysts initially expressed markers for the inner cell mass, epiblast and primitive endoderm. After 1-2 days in culture, a trophoblast marker appeared, and epiblast, primitive endoderm and trophoblast fates were well defined. Then the epiblast started to polarize and the primary yolk sac developed, followed by the secondary yolk

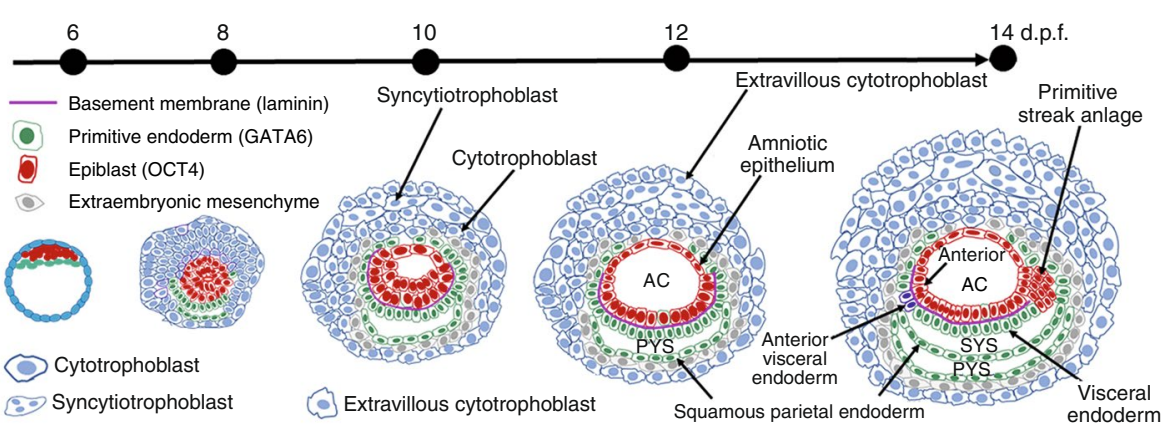

Schematic overview of early human development. AC, amniotic cavity; PYS, primary yolk sac; SYS, secondary yolk sac; d.p.f., days post-fertilization; GATA6 and OCT4, transcription factors. Adapted with permission from Xiang, L. et al. A developmental landscape of 3D-cultured human pre-gastrulation embryos. Nature https://doi.org/10.1038/s41586-019-1875-y (2019).

sac. Moreover, a clear difference between the amniotic epithelium and the epiblast arose, which had not been observed in embryos cultured under 2D conditions. Additional features that had previously not been observed were the appearance of anteriorposterior polarity in the embryo and the formation of the primitive streak anlage.

Having a suitable model for human embryogenesis in hand allows $\mathrm{Li}$, Ji and their teams "to systematically reveal the molecular and morphogenetic developmental landscape of pregastrulation human embryos [and to] delineate specific pathways and transcription factors for epiblast, amniotic epithelium, hypoblast and trophoblast separation and development." To do so, the researchers performed single-cell RNA-seq experiments on seven different stages of embryogenesis, which allowed them to identify additional markers for embryonic tissues and to describe crucial signaling pathways involved in the various developmental processes, such as amniotic epithelium emergence and trophoblast differentiation.

Furthermore, the transcriptomic analysis revealed key differences between human and macaque embryos in, for example, the epiblast, where bone morphogenetic protein (BMP) signaling patterns differed between the two species. "Although monkey has a high similarity with human, these differences indicate monkey embryos cannot completely replace human embryos in uncovering human development, and human embryo studies are very necessary," says Li. This is one of the reasons why Li thinks that - after a thorough ethical discussion and evaluation by the international community - extending human embryo culture beyond the internationally agreed upon 14-day limit is necessary for a better understanding of events during human gastrulation.

In the meantime, $\mathrm{Li}$ and his colleagues plan to follow up on their work. They want to understand why three-quarters of embryos fail to develop in culture. In addition, they want to gain a deeper understanding of the mechanisms of human embryo development. Current studies have only scratched the surface. This is an exciting time for understanding how our species develops.

Nina Vogt

Published online: 4 February 2020 https://doi.org/10.1038/s41592-020-0740-0

Research paper

Xiang, L. et al. A developmental landscape of 3D-cultured human pre-gastrulation embryos. Nature https://doi.org/10.1038/s41586-019-1875-y (2019). 